\title{
CFD Simulation of a Horizontal Axis Hydrokinetic Turbine
}

\author{
L.T. Contreras ${ }^{1}$, Y.U. López ${ }^{2}$ and S. Laín ${ }^{1}$ \\ ${ }^{1}$ Modelling, Analysis \& Simulation of Environmental \& Industrial Processes (PAI+) Research Group \\ ${ }^{2}$ Energy Research Group \\ Energetics and Mechanics Department, Faculty of Engineering, Universidad Autónoma de Occidente \\ Campus Valle del Lili - Calle 25 No 115-85, Cali (Colombia)
}

Phone:+0057 2318 8000, e-mail: Itcontreras@uao.edu.co,ylopez@uao.edu.co, santiago.lain@gmail.com

\begin{abstract}
This study presents three-dimensional full transient numerical simulations of a horizontal axis hydrokinetic turbine, HAHT with particular emphasis on the analysis of its hydrodynamic characteristics. Hydrokinetic turbine performance is studied using a time-accurate Reynolds-averaged NavierStokes (RANS) commercial solver. A physical transient rotorstator model with a sliding mesh technique is used to capture changes in flow field at a particular time step. A shear stress transport (SST) turbulence model has been employed to model the turbulent features of the flow. The studied rotor has three blades, based on NACA4412 airfoil. Two operation conditions have been considered: shaft parallel to the incoming flow (SP configuration) and shaft inclined an angle $\alpha$ around $30^{\circ}$ regarding the main stream (SI configuration). As a result, the decrement of the hydrodynamic performance of the turbine with the inclined axis is quantitatively evaluated regarding that of the parallel axis. Moreover, a preliminary study of the vorticity dynamics in the wake of the inclined rotor is performed
\end{abstract}

\section{Key words}

CFD simulation, transient analysis, inclined axis hydrokinetic turbine, turbulence model.

\section{Introduction}

Kinetic energy available in the water currents has generated a great attraction due its high energy density, high predictability and low environmental impacts (minimal visual impact, no emission and no noise). Energy can be extracted from ocean and river currents by using submerged turbines, which are similar in function to wind turbines, capturing energy through the processes of hydrodynamic, rather than aerodynamic, lift or drag. Hydrokinetic energy does not require reservoirs or dams and does not generate emissions. Hence, environmental impact is minimal, and the site selection is much less restrictive compared with other hydro-technologies. The initial installation costs and the implementation time is relatively short because this type of energy generation in the rivers does not require a significant infrastructure such as dams. The modular nature of hydraulic power leads to an easily scalable output energy, with opportunities for reducing the cost per $\mathrm{kW}$. Moreover, as water in the ocean or river flows almost constantly there is a continuous power generation, thus eliminating the need of any energy storage, which is an important advantage for the isolated communities and interesting in public services.

The study of flow dynamics in hydrokinetic turbines has had a great interest in recent years by the scientific community, particularly in relation to improving efficiencies and understanding of their performance. However, it still requires a deeply understanding of its performance especially for hydrodynamic applications. Computational Fluid Dynamics (CFD) has proven to be a useful tool for flow analysis around this type of turbines. For example regarding performance prediction of horizontal axis water turbines, $\mathrm{Wu}$ et al [1] shows numerical results for the power coefficient $\left(C_{p}\right)$ using CFD that are in satisfactory agreement with experimental data. In the present study, the software ANSYS - Fluent has been employed to perform the numerical simulation of the horizontal axis hydrokinetic turbine (HAHT).

This work focuses on the performance of small horizontal axis hydrokinetic turbines, of Garman type. Such turbines are mounted on a floating device on a river or channel and operate with its shaft inclined respect to the main flow direction (see Fig.1 top). The design of these devices has been mainly empirical, so only few experimental studies are available [2, 5]. For example, [2] conducted experimental measurements of a threeblade Garman turbine in a laboratory channel whose axis was inclined $45^{\circ}$ regarding the main stream for three different incoming flow speeds. Regarding the performance, an average mechanical system efficiency of $30 \%$ was reported. However, nothing was found about numerical CFD simulation of such configuration. Therefore, as far as the authors are aware, this is the first time in which an inclined configuration of a horizontal axis hydrokinetic turbine is attempted to be simulated by using CFD. 


\section{Turbine Configuration}

The horizontal axis hydrokinetic turbine has been chosen based on an existing machine named Aquavatio, which was constructed empirically, modifying a former design of a Garman type machine. The rotor diameter D is $1.8 \mathrm{~m}$, the blades are based on the NACA 4412 airfoil with a lineal distribution of both, twist and chord which decreases towards the blade tip as usual. Connection of blades to shaft is performed by means of a truncated conical hub. Fig. 1 (bottom) shows an isometric view of the turbine assembly, where the flow is aligned with the $\mathrm{z}$ axis; $\mathrm{y}$ denotes the vertical axis and $\mathrm{x}$ the lateral axis forming a right-handed coordinate system.
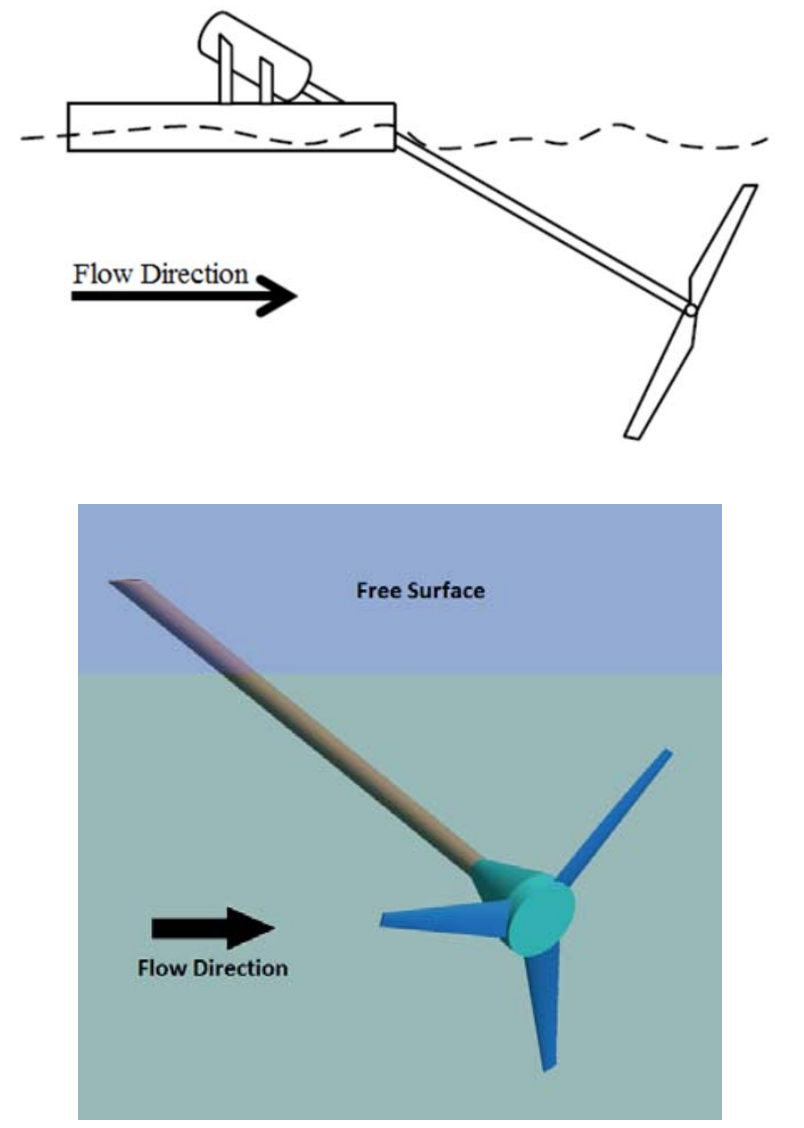

Fig. 1. Schematics of the geometry considered for the hydrokinetic turbine (top) and geometrical model considered in isometric view (bottom).

Geometry employed in simulation is a three-dimensional model of real turbine. Moreover, the shaft was not included in the simulation of the parallel to flow configuration, but it was included in the inclined turbine configuration. Dimensions of computational domain are: length, $38.5 \mathrm{R}$; width, $13 \mathrm{D}$; height, $11 \mathrm{R}$, where $\mathrm{R}$ is the rotor radius. Such dimensions guarantee that the turbine can be considered to operate almost isolated. Computational domain consists of two sub-domains: a cylindrical inner domain, containing the hub and the blades; and an external steady domain representing the free stream. In order to reproduce the transient behaviour of the rotating blades the cylindrical domain rotates respect the steady domain with a prescribed angular velocity. The coupling between both sub-domains is performed by means of the sliding mesh method, available in the employed software (ANSYS-Fluent v. 14).
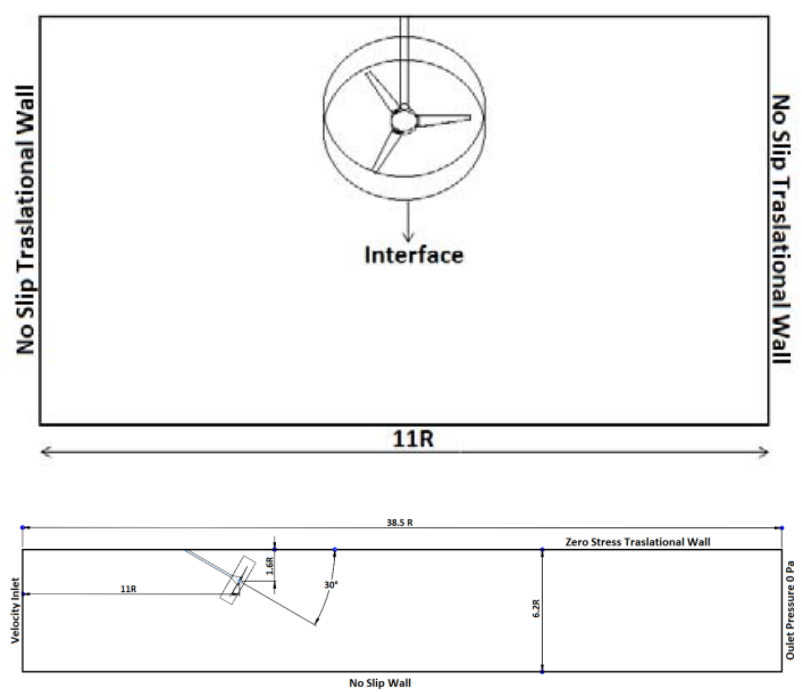

Fig. 2. Front view (top) and lateral view (bottom) of the geometry for the inclined hydrokinetic turbine, showing the boundary conditions employed.

Following the standard notation of CFD, boundary conditions employed in the computations (Fig. 2) consist of a velocity inlet on bottom side (constant equal to 1.0 $\mathrm{m} / \mathrm{s})$, a pressure outlet on top side ( $0 \mathrm{~Pa}$ ) and non-slip translational walls on west and east boundaries; north side was specified as a zero stress translational wall representing the free surface, while the south boundary is specified as a non-slip wall. The blades are in the inner part of a rotating cylinder, which is separated from the steady domain by sliding interfaces specified as a boundary condition of type sliding mesh. In case of the hydrokinetic turbine, the computational domain consists of a rotating zone (rotor in a cylindrical domain) and a steady zone, which includes water environment outside of the cylindrical domain. Mesh closest to profiles must be refined to describe with sufficient precision the boundary layer flow. Created mesh had an O-grid topology based on prisms. Outside of this prism layer, a non-structured grid based on tetras was chosen, keeping an aspect ratio similar to that of prisms (see Fig. 3 top). The employed number of prism layers in this work is 12 . Additionally, Fig. 3 (bottom) shows a detail of the surface mesh of the rotor.

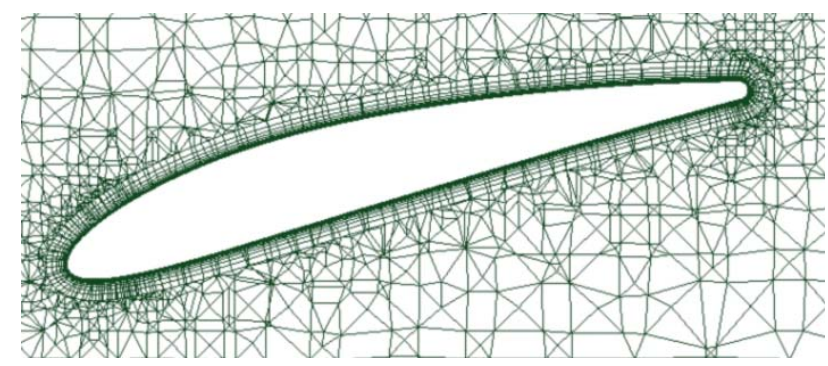




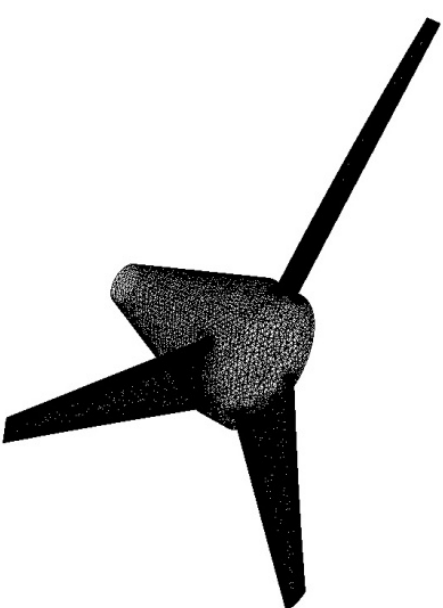

Fig. 3. Detail of the prism layers around the blade profile (top) and of thesurface mesh on the rotor (blades and hub) (bottom).

Steady domain was also discretized with a non-structured grid based on tetras. Grid node density is higher near blades than in rest of the domain. Moreover, due to complexity of flow in turbine wake, also grid node density is higher downstream than upstream. The most interesting zone for simulation is the cylindrical domain, where flow interacts with blades, which is responsible for turbine performance. Again, in this region, the grid node density is higher than in the steady domain.

In this paper, two rotor configurations are considered: the standard configuration in which the shaft is parallel to main flow (SP configuration) and a second one where the shaft makes an angle of $30^{\circ}$ regarding the free surface (SI configuration). The motivation for simulating the second arrangement is that the prototype operates in a similar way. Moreover, although there are some experimental studies [2], as far as the authors are aware, this is the first time in which an inclined configuration of a horizontal axis hydrokinetic turbine (HAHT) is attempted to be simulated by using CFD.

\section{Simulation Set-up}

Numerical simulations were carried out using the commercial CFD software ANSYS FLUENT v14.0. A sliding mesh scheme was employed to simulate the rotation of the blades at a constant angular velocity of 60 rpm, which allows capturing the change of the flow field at a particular time. The Shear Stress Transport (SST) turbulence model was used to model the turbulent features of the flow [6-8]. This method is a combination of the $k-\varepsilon$ and $k-\omega$ models [3]: it uses the k- $\omega$ model near the wall and switches to a function of the k- $\varepsilon$ model when moving away from the wall closer to the upper limit of the boundary layer. The SST model has been shown to give good performance for flows with strong adverse pressure gradients such as those appearing in the horizontal axis hydrokinetic turbine flow configuration.

The computations were carried out in a mesh of about 7-8 millions of elements although some simulations were also run in a finer mesh of around 11 million. In that case the differences on turbine efficiency are lower than $1 \%$. The mesh size in each of the configurations is very similar. During meshing, special care was taken to build a prism layer around the blades' wall of high quality (i.e. smooth transition between the layers and low element skewness), guarantying in all the cases values of $\mathrm{y}^{+}$in linear sublayer (i.e., below 5). Such constraint is required by the employed turbulence model and also it is needed for the correct description of the flow dynamics around the blades [4]. Moreover, all the results where converged using second order schemes, in time as well as in space for all the variables. For instance the variation of the torque between successive turns was below $0.2 \%$. Consequently, the obtained results are robust from a numerical point of view. The computations were performed for an angular velocity of $60 \mathrm{rpm}$ at tip speed ratio $\lambda=5.736$ using a time step equal to $5 \mathrm{~ms}$.

Typically, the simulation starts with the computation of the steady flow around a fixed position of the turbine blades. From this initial condition, the transient simulation begins, firstly with first order schemes to ease convergence. Once that the total torque on the turbine has reached a quasi-periodic regime, after three or four complete rotor revolutions, the discretization schemes are switched to second order. Finally, the simulation runs during a sufficient number or rotor revolutions in the quasi-periodic regime to extract an average value for the torque, which is used to estimate the turbine performance.

\section{Results and Discussion}

In order to analyze the numerical performance of the turbine, the following non-dimensional parameters have to be defined: tip speed ratio $\lambda$ which is defined by equation (1) and represents the non-dimensional angular velocity.

$\lambda=\frac{\omega R}{V_{0}}$

Where $\omega$ represents turbine angular velocity, $\mathrm{R}$ the rotor radius and $V_{0}$ the freestream velocity. The torque $M$ produced by the turbine in dimensionless form is given by the moment coefficient $\left(\mathrm{C}_{\mathrm{m}}\right)$.

$$
\mathrm{C}_{\mathrm{m}}=\frac{\mathrm{M}}{\frac{1}{2} \rho \mathrm{V}_{0}{ }^{2} \mathrm{RS}_{\mathrm{ref}}}
$$

Where $\rho$ is the water density and $S_{\text {ref }}$ is the area swept by the rotor $\left(\mathrm{S}_{\mathrm{ref}}=\pi R^{2}\right)$. The power $(\mathrm{P})$ produced by the turbine in dimensionless form is defined by the power coefficient $\left(C_{P}\right)$, which is also its efficiency.

$\mathrm{C}_{\mathrm{p}}=\frac{\mathrm{P}}{\frac{1}{2} \rho \mathrm{V}_{0}{ }^{3} \mathrm{~S}_{\mathrm{ref}}}$

The resultant aerodynamic force acting on the blades is decomposed into a normal force which is perpendicular to the rotor $\left(\mathrm{F}_{\mathrm{n}}\right)$ and a tangential force $\left(\mathrm{F}_{\mathrm{t}}\right)$ parallel to rotation plane. The tangential force coefficient $\left(C_{t}\right)$ is 
related with the torque produced by the fluid on the turbine. A negative value indicates torque generation by the turbine. The normal force coefficient $\left(C_{n}\right)$ is related to cyclic loading and fatigue on the blades and it is also referred as thrust coefficient

$$
\begin{aligned}
C_{n} & =\frac{F_{n}}{\frac{1}{2} \rho V_{0}^{2} S_{r e f}} \\
C_{t} & =\frac{F_{t}}{\frac{1}{2} \rho V_{0}^{2} S_{r e f}}
\end{aligned}
$$

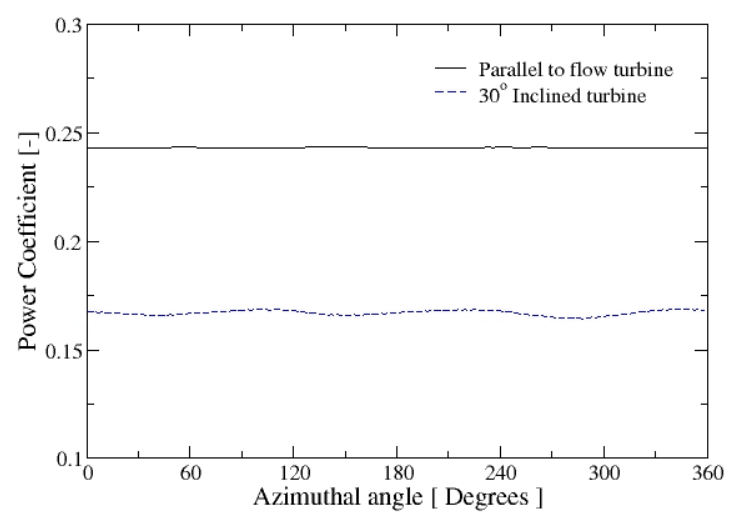

Fig. 4. Power coefficient at $\lambda=5.736$ for the two studied configurations, parallel and $30^{\circ}$ inclined respect the flow direction.

Power coefficient $C_{p}$ values obtained for the two configurations, SP and SI, are presented in Fig. 4. In such figure, the evolution of $C_{p}$ along the azimuthal angle, for a turbine revolution, presents a quasi-periodic behavior, which indicates that the initial transient has been overcome.

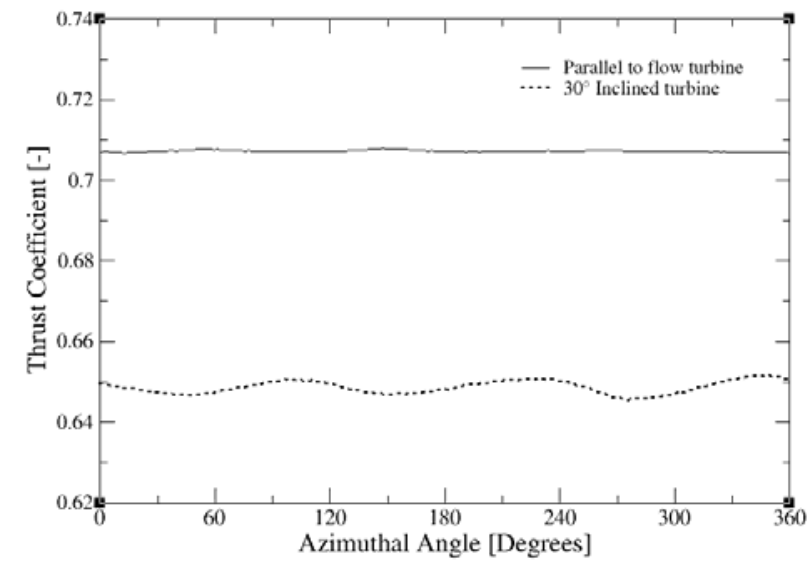

Fig. 5. Thrust coefficient at $\lambda=5.736$ for the two studied configurations, parallel and $30^{\circ}$ inclined respect the flow direction.

From the results shown in Fig. 4, the $C_{p}$ curve of the inclined turbine shows a gentle ripple in contrast to the nearly straight line obtained for the parallel turbine. This fact is due to the differences in the incoming velocity seen by the turbine blades along a revolution in the inclined turbine case. As expected the power coefficient in this case is reduced nearly a $30 \%$ regarding the parallel configuration. It is necessary to point out that the small efficiency value of the SP configuration, lower than 25\%, is due to the truncated conical hub geometry, which generates a wide wake responsible also for increasing the drag on the turbine (see Fig. 8).

Thrust coefficient, given by Eq. (4), is illustrated in Fig. 5 versus azimuthal angle along a turbine revolution. As it can be readily seen, the normal force coefficient is remarkably lower in the case of the inclined axis turbine than for the parallel configuration, roughly 8\% lower. However, in the former case the blades are subjected to alternating stresses that could induce material fatigue. Such alternating normal forces are absent in the parallel configuration as in this case the load on the blades is approximately constant in a revolution (see Fig. 5).

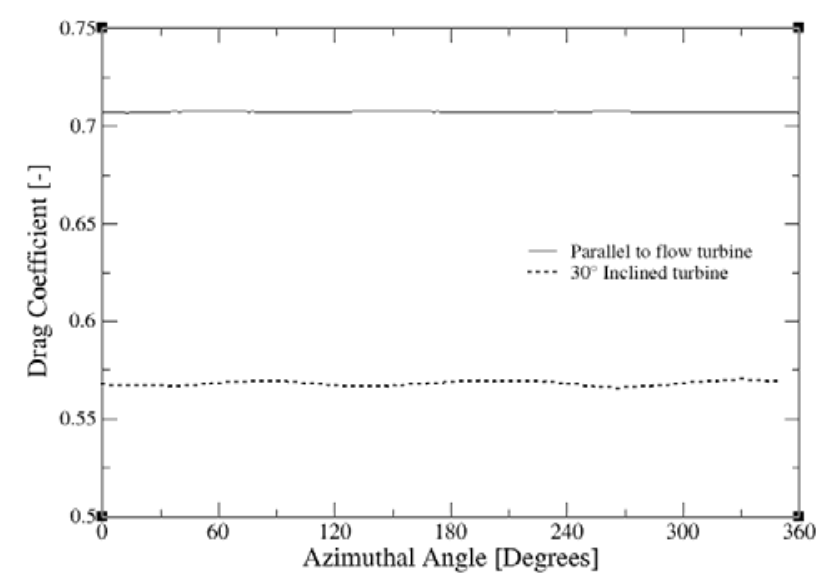

Fig. 6. Drag coefficient at $\lambda=5.736$ for the two studied configurations, parallel and $30^{\circ}$ inclined respect the flow direction.

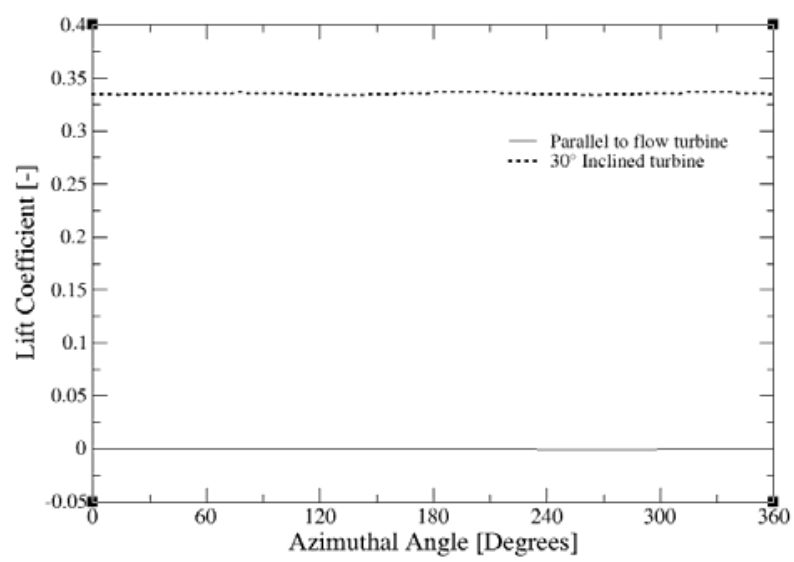

Fig. 7. Lift coefficient at $\lambda=5.736$ for the two studied configurations, parallel and $30^{\circ}$ inclined respect the flow direction.

Another way of analysing the forces on a submerged body is to decompose the total experienced force on parallel to flow component, i.e. the drag force, and perpendicular to flow component, i.e., the lift force. The 
corresponding coefficients are shown in Figs. 6 and 7, respectively.

In Fig. 6 it can be observed that the drag coefficient of the inclined turbine is around a $20 \%$ lower than that of the parallel configuration, in accordance with the former results. This is of course due to the high contribution of pressure drag in the case of the perpendicular rotor. On the other hand, as it is seen in Fig. 7, in the inclined configuration the turbine experiences a net upward lift force which is absent in the first arrangement. Composing both components to obtain the total force acting on the rotor, it is found that the loads on the inclined turbine are smaller than on the parallel turbine, which is related to the larger wake dimensions in this last case.
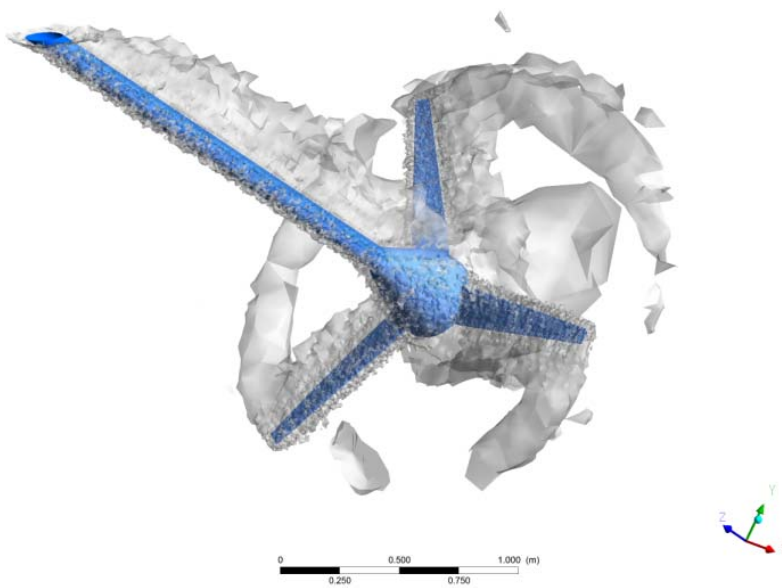

Fig. 8. Vorticity isosurface showing the detached boundary layer in the turbine shaft and the blades. Tip vortices are clearly visible.

In order to illustrate the detached trailing vortices from blades tip in the case of inclined turbine, Fig. 8 shows a vorticity isosurface. Also, the wake behind the shaft can be clearly appreciated as well as the wake behind the hub. This last one is characterized by the presence of a quasisteady toroidal vortex inducing a wide zone of backward flow that increases drag and thrust forces on the rotor and also reduce turbine performance.

Finally, Fig. 9 shows that the laminar to turbulent boundary layer transition phenomenon in the first blade of the hydrokinetic turbine by means of the intermittency function $\gamma$, computed using the transition version of the SST turbulence model. $\gamma$ values close to 0 indicate laminar flow (black colour in the plots). The upper plot deals with the parallel configuration and the lower plot with the inclined turbine. In both cases the blade is located at the same azimuthal angle. As it can be readily seen, both contour plots are quite different showing in the first case that the boundary layer in the intrados of the profile develops in the laminar regime along the whole chord. In the inclined turbine case transition from the laminar to turbulent regime are observed for both surfaces, intrados and extrados.

The last two figures illustrate the complexity of the water flow around the turbine blades of the horizontal axis hydrokinetic turbine, which will be subject of more detailed study in the near future.

\section{Conclusion}

The present paper presents for first time the threedimensional unsteady CFD simulation of a horizontal axis hydrokinetic turbine operating with an inclined shaft respect to the incoming flow. The obtained results allowed quantifying the reduction on performance regarding the parallel to flow configuration. In the considered case such reduction was about 30\%. Moreover, it has also been demonstrated that the inclined configuration is exposed to lower thrust and drag forces than the perpendicular to flow arrangement, although in the first case the blades experience alternating stresses that may induce in the long term failure due to fatigue.
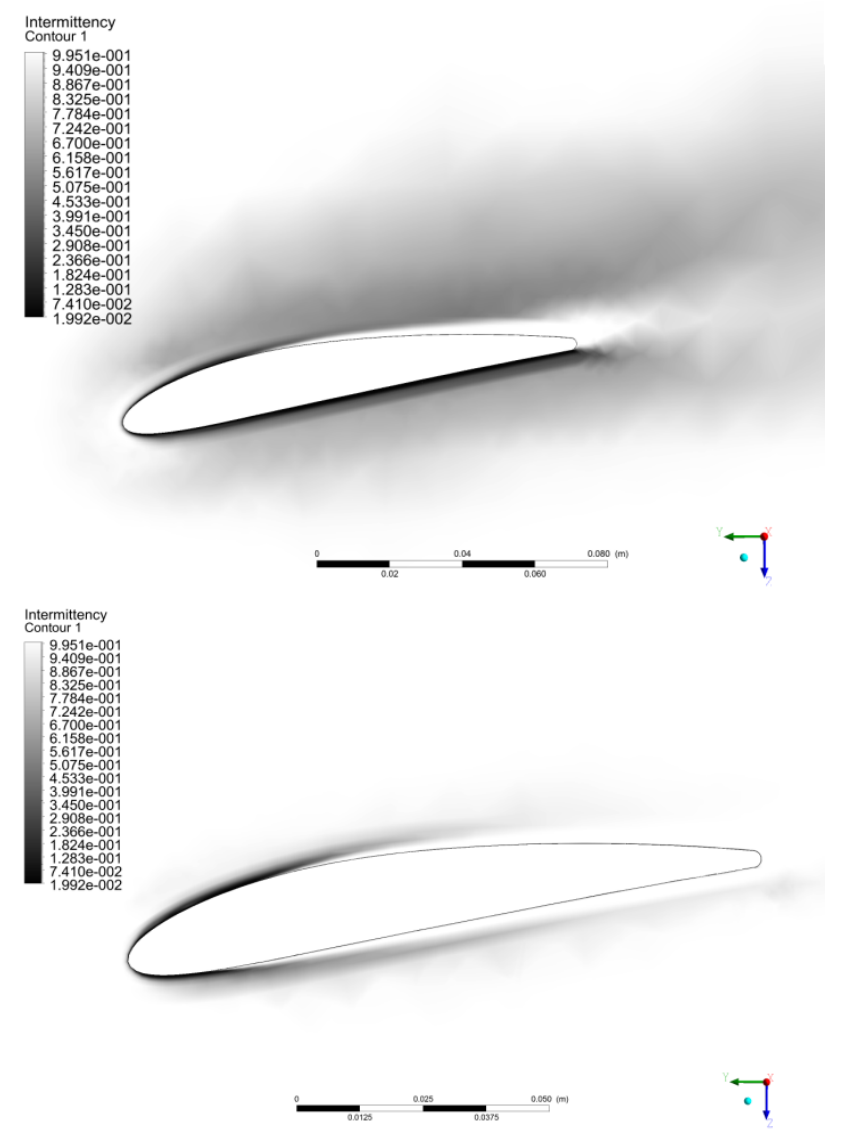

Fig. 9. Intermittency contours around the first blade. Top: parallel to flow configuration. Bottom: $30^{\circ}$ inclined turbine. Black colour indicates laminar flow.

\section{Acknowledgement}

The financial support of the Dirección de Investigaciones y Desarrollo Tecnológico of Universidad Autónoma de Occidente is gratefully acknowledged. This work was partially sponsored by the Young Researchers program from the Colombian administrative department of science, technology and innovation, Colciencias (L.T. Contreras). 


\section{References}

[1] Wu, H., Chen, L., Yu, M., Li, W., Chen, B. "On design and performance prediction of the horizontal axis water turbine". Ocean Engineering, Vol. 50 (2012), pp. 23-30.

[2] Al Mamun. N.H. "Utilization of river current for small scale electricity generation in Bangladesh”. M.Sc. Thesis, University of Bangladesh (2001).

[3] Menter, F. R. "Two-Equation Eddy-Viscosity Turbulence Models for Engineering Applications”. AIAA. J., vol. 32, 269-289 (1994).

[4] Maître, T., Amet, E., Pellone, C. "Modeling of the flow in a Darrieus water turbine: Wall grid refinement analysis and comparison with experiments”. Renewable Energy, vol. 51, 497 - 512 (2013).
[5] Islam, A. S., Al-Mamun, N. H., Islam, M. Q., Infield, D. G. Energy from river current for small scale electricity generation in Bangladesh. In INTERNATIONAL SOLAR ENERGY SOCIETY UK SECTION-CONFERENCE-C pp. 207-213 (2001).

[6] S. Laín, O. López, B. Quintero and D. Meneses, "Design Optimization of a Vertical Axis Water Turbine with CFD," in Alternative Energies - Advanced Structured Material, Berlin Heidelberg, Springer, 2013, pp. 113-139.

[7] D. Meneses, O. López, B. Quintero and S. Lain, "Computational study of transient flow around Darrieus type cross flow water turbines," Journal of Renewable and Sustainable Energy, vol. 8, no. 014501, 2016.

[8] Y. Dai and W. Lam, "Numerical study of straight-bladed Darrieus-Type tidal turbine," ICE Energy, vol. 162, pp. 67-76, 2009. 